\title{
On the dynamical state of galaxy clusters: insights from cosmological simulations II.
}

\author{
Weiguang Cui, ${ }^{1,2 \star}$ Chris Power, ${ }^{1,2}$ Stefano Borgani, ${ }^{3,4,5}$ Alexander Knebe, ${ }^{6,7}$ Geraint \\ F. Lewis, ${ }^{8}$ Giuseppe Murante, ${ }^{3}$ and Gregory B. Poole, ${ }^{9}$ \\ ${ }^{1}$ ICRAR, University of Western Australia, 35 Stirling Highway, Crawley, Western Australia 6009, Australia \\ 2 ARC Centre of Excellence for All-Sky Astrophysics (CAASTRO) \\ 3 Astronomy Unit, Department of Physics, University of Trieste, via Tiepolo 11, I-34131 Trieste, Italy \\ 4 INAF - Astronomical Observatory of Trieste, via Tiepolo 11, I-34131 Trieste, Italy \\ ${ }^{5}$ INFN - Sezione di Trieste, I-34100 Trieste, Italy \\ ${ }^{6}$ Departamento de Física Teórica, Módulo 15, Facultad de Ciencias, Universidad Autónoma de Madrid, 28049 Madrid, Spain \\ ${ }^{7}$ Astro-UAM, UAM, Unidad Asociada CSIC \\ 8 Sydney Institute for Astronomy, School of Physics, A28, The University of Sydney NSW 2006, Australia \\ 9 School of Physics, University of Melbourne, Parksville, VIC 3010, Australia
}

Accepted XXX. Received YYY; in original form ZZZ

\begin{abstract}
Using a suite of cosmology simulations of a sample of $>120$ galaxy clusters with $\log \left(M_{D M, v i r}\right) \leqslant 14.5$. We compare clusters that form in purely dark matter run and their counterparts in hydro runs and investigate 4 independent parameters, that are normally used to classify dynamical state. We find that the virial ratio $\eta$ in hydrodynamical runs is $\sim 10$ per cent lower than in the DM run, and there is no clear separation between the relaxed and unrelaxed clusters for any parameter. Further, using the velocity dispersion deviation parameter $\zeta$, which is defined as the ratio between cluster velocity dispersion $\sigma$ and the theoretical prediction $\sigma_{t}=\sqrt{G M_{\text {total }} / R}$, we find that there is a linear correlation between the virial ratio $\eta$ and this $\zeta$ parameter. We propose to use this $\zeta$ parameter, which can be easily derived from observed galaxy clusters, as a substitute of the $\eta$ parameter to quantify the cluster dynamical state.
\end{abstract}

Key words: galaxies: clusters: general - galaxies: kinematics and dynamics - galaxies: halos - galaxies: evolution - cosmology: theory

\section{INTRODUCTION}

Currently favored models of cosmological structure formation are hierarchical - lower mass systems merge progressively to form more massive structures, with galaxy clusters representing the final state of this process. The dynamical process, driven by gravity, determines the final properties of the dark matter halo, as well as the baryonic contents in it galaxies, intra cluster medium (ICM), etc. However, even at the final state of hierarchical structure formation, the galaxy clusters are not always in dynamic equilibrium. In observations, galaxy cluster systems can be roughly separated into relaxed and unrelaxed; the ICM in relaxed clusters is normally in hydrostatic equilibrium, while dynamically unrelaxed clusters are undergoing, or have undergone, a merger, which leaves the ICM turbulent (see Wen \& Han 2013, and references therein). In simulations, there are a vague of ways dynamical state can be evaluated.

* E-mail: weiguang.cui@uwa.edu.au
Using dark-matter-only simulations, Jing (2000) found that about 30 per cent of the simulated dark matter halos can not be fitted by the NFW profile (Navarro et al. 1997), and these halos that showed larger deviations from the NFW profile exhibited significant internal substructures. Using the integral virial ratio parameter $2 T /|W|+1$, here $\mathrm{T}$ is the kinetic energy, $\mathrm{W}$ is the potential energy, Bett et al. (2007) suggested $2 T / W+1<1.5$ to select halos in quasiequilibrium states (see also Klypin et al. 2016). Neto et al. (2007) expanded the criteria by including substructure mass fraction and centre-of-mass offset. However, they adopted a narrower limit for their virial ratio $2 T /|W|<1.35$ (see also Ludlow et al. 2012). Shaw et al. (2006); Poole et al. (2006); Davis et al. (2011) modified the virial ratio by taking the surface pressure energy $E_{s}$ into account. This is because halos are not isolated in cosmology simulations, and infalling materials alter $2 T / W$. Besides the surface pressure energy, Davis et al. (2011) also considered the potential energy from particles outside of halos $-W_{\text {ext }}$ for the virial ratio. However, they found that $W_{\text {ext }}$ is negligible. Nevertheless, different 
limits are used to calculate the virial ratio: $\left(2 T-E_{s}\right) / W+$ $1>-0.2$ for Shaw et al. (2006); $\left|1+2 T /\left(E_{s}+W\right)\right|<0.02$ for Poole et al. (2006); While Knebe \& Power (2008) suggested $-0.15 \leqslant\left(2 T-E_{s}\right) / W+1 \leqslant 0.15$ (with a mass dependence at $z=1$ ) to select out relaxed halos. Power et al. (2012) studied the relation between centre-of-mass offset and equilibrium state. Instead of using virial ratio, they suggested a centre of mass offset value of 0.04 to select relaxed halos.

All of these studies were based on dark matter only simulations. However, as numerical simulations with sophisticated sub-grid baryon models have become more mature and successful in producing observed-like galaxies, there has been great interest in studying the baryonic effects on galaxy cluster properties (e.g. Schaller et al. 2015; Cui et al. 2016a); on power spectrum (e.g. van Daalen et al. 2011); on halo mass as well as halo mass function (e.g. Cui et al. 2012, 2014b; Velliscig et al. 2014); and on substructure shapes and alignments (e.g. Knebe et al. 2010; Velliscig et al. 2015). It is timely and interesting to study and how baryons affect the dynamical state of galaxy clusters. Baryons, especially gas, are subject to other forces in addition to gravity to dark matter, which will lead changes on $\mathrm{T}$ and $\mathrm{W}$.

In this paper, we study the dynamical state of galaxy clusters with a volume- and mass-complete sample from a series of cosmological simulations with three different baryon models, which we have presented in Cui et al. (2016b, hereafter Paper I). We investigate how different measures of dynamical state change between dark-matter-only and hydrodynamical runs.

In the following sections, we briefly describe these hydro-simulations with different baryon models (see also Cui et al. 2012, 2014b) and the statistical sample of clusters (see also Paper I) (§ 2), and present our dynamical state classification methods $(\S 3)$. In section 4 we present our results. Finally, we summarise our conclusions in $\S 5$, and comment on the implications for interpretation of observations of galaxy clusters.

\section{SIMULATED GALAXY CLUSTER CATALOGUE}

These simulations use a flat $\Lambda$ CDM cosmology, with cosmological parameters of $\Omega_{\mathrm{m}}=0.24$ for the matter density parameter, $\Omega_{\mathrm{b}}=0.0413$ for the baryon contribution, $\sigma_{8}=0.8$ for the power spectrum normalization, $n_{\mathrm{s}}=0.96$ for the primordial spectral index, and $h=0.73$ for the Hubble parameter in units of $100 h \mathrm{~km} \mathrm{~s}^{-1} \mathrm{Mpc}^{-1}$. They used the same realization of the initial matter power spectrum, and were run with the TreePM-SPH code GADGET-3, an improved version of the public GADGET-2 code (Springel 2005). Three simulations were run, we refer to the darkmatter-only simulation as the DM run; the hydrodynamical simulations including radiative cooling, star formation and kinetic feedback from supernovae: in one case we ignore feedback from AGN (which is referred as the CSF run), while in the other we include it (which is referred as the AGN run). The DM run has two families of dark matter particles: the one with larger particle mass shares the same ID as the dark matter particles in the CSF and AGN runs; while the one with smaller particle mass has equal mass as the gas particles in the CSF and AGN runs at the initial condition of $z$
$=49$. With this particular setup, we can make an explicated investigation on the baryon effect.

Halos are identified using the Python spherIcAl Overdensity (SO) algorithm PIAO ${ }^{1}$ (Cui et al. 2014b), and are selected from the DM run with a mass cut. We reselect 123 halos, which have the virial mass of $\log _{10}\left(M_{v i r}\right)>$ $14.5 h^{-1} \mathrm{M}_{\odot}$. We use Bryan \& Norman (1998) t oestimate $\Delta_{v i r}$ and compute $M_{v i r}$. Counter parts SO halos in AGN and CSF runs are identified by cross-matching dark matter components using their unique particle IDs (also see Cui et al. 2014b, more details).

\section{METHODS}

Virial Ratio The exact virial theorem for a self-gravitating system is

$$
\frac{1}{2} \frac{d^{2} I}{d t^{2}}=2 T+W-E_{s}
$$

where I is the moment of inertia. The proper way of calculating the equation 1 is by using the time averaged values of these quantities (see discussion in Poole et al. 2006). However, due to the limited outputs of the simulation, we only calculate these quantities at $z=0$.

Total kinetic energy $\mathrm{T}$ is calculated differently for collisionless (dark matter and star) particles and collisional (gas) particles. After removing the halo motion, which is given by the mass-weighted mean velocity from particles within $30 \mathrm{kpc}$, and the Hubble flow, $\mathrm{T}$ is simply $\frac{1}{2} m_{i} v_{i}^{2}$, where $i$ is for all collisionless particles; We use the gas thermal energy $\mathrm{U}$ for its kinetic energy. Total potential energy $\mathrm{W}$ is directly calculated by using all particles inside halos without any approximation. $E_{s}$ is the energy from surface pressure $\mathrm{P}$ at the halo boundary. As described in Chandrasekhar (1961), $E_{s}$ is

$E_{s}=\int P(r) \boldsymbol{r} \cdot \boldsymbol{d} \boldsymbol{S}$.

Assuming the ideal gas law, P for collisionless particles (see Shaw et al. 2006, for more details) can be written as

$P_{c}=\frac{\sum_{i} m_{i} v_{i}^{2}}{3 V}$

this summation is over all particles with mass $m_{i}$, velocity $v_{i}$ inside volume $\mathrm{V}$, while $\mathrm{P}$ for gas particles (see Poole et al. 2006, for more details) is

$P_{g}=\frac{\sum_{i} N_{i} k_{B} T_{i}}{V}$

here $N_{i}, T_{i}$ are the gas number and temperature respectively, $k_{B}$ is the Boltzmann constant.

We follow Shaw et al. (2006) to calculate P: first, we rank order all particles by their radius and select the outermost 20 per cent; then we label the radius of the innermost particle in this shell as $R_{0.8}$, the outermost as $R_{v i r}$, and the median as $R_{0.9} . \mathrm{V}$ is the volume occupied by the outermost 20 per cent particles, $V=\frac{4 \pi}{3}\left(R_{v i r}^{3}-R_{0.8}^{3}\right)$. The surface pressure energy from collisionless component can be approximated by

$E_{s, c} \approx 4 \pi R_{0.9}^{3} P_{c}=\frac{R_{0.9}^{3}}{R_{v i r}^{3}-R_{0.8}^{3}} \sum_{i} m_{i} v_{i}^{2}$.

${ }^{1}$ It is publicly available at https://github.com/ilaudy/PIAO 
For gaseous particles, the gas number density $\mathrm{n}$ can be expressed in terms of the mean molecular weight: $\mu=$ $\rho /\left(n m_{p}\right)$, where $m_{p}$ is the mass of a proton, $\rho$ is gas density. Following Mo et al. (2010), we assume the elements heavier than helium have a mass number $M_{i} \approx 2\left(Q_{i}+1\right)$, here $Q_{i}+1$ is the charge number of a fully ionized atom. If we define the total mass as $X_{i}=1$, where $X_{i}$ is the mass abundance of element $\mathrm{i}$, then we have $\mu=4 /\left(6 X_{H}+X_{H e}+2\right)$. Normally we assume the metallicity $Z=1-X_{H}-X_{H e}$ is very small, and the mass fraction for hydrogen is around 0.76 . Thus, we can have $\mu \approx 0.588$ and the gas number

$N=n V=\frac{\rho V}{\mu m_{p}}=\frac{m}{\mu m_{p}}$.

Finally, we can calculate the surface pressure energy from the gas component as,

$E_{s, g} \approx 4 \pi R_{0.9}^{3} P_{g}=\frac{R_{0.9}^{3}}{R_{v i r}^{3}-R_{0.8}^{3}} \frac{3 k_{B}}{\mu m_{p}} \sum_{i} m_{i} T_{i}$,

where summation is over all the gas particles lying between $R_{0.8}$ and $R_{v i r}$. $E_{s}$ is contributed by both collisionless and gas particles.

If the system is in a steady state and dynamical equilibrium, equation (1) will reduce to $2 T+W-E_{s}=0$, which can be rewritten as $\left(2 T-E_{s}\right) /|W|=1$. Therefore, we define $\eta=\left(2 T-E_{s}\right) /|W|$, and expect $\eta \rightarrow 1$ for dynamically relaxed galaxy clusters.

Total subhalo mass fraction Subhalos are identified by SubFind (Springel et al. 2001; Dolag et al. 2009; Cui et al. 2014a). For all the galaxy clusters identified by PIAO, we run SubFind on them one by one. The smallest subhalo has at least 32 particles. Subhalos with only gas particles are not taken into account (Dolag et al. 2009). The subhalo mass fraction $f_{s}$ does not include the most massive substructure as this is simply the bound component of the main halo.

Centre-of-mass offset For all the particles within the virial radius $R_{v i r}$, we compute the centre of mass as

$\boldsymbol{R}_{c m}=\frac{1}{M} \sum_{i=1}^{n} m_{i} r_{i}$,

here $m_{i}$ is the $i^{t h}$ particle mass, $r_{i}$ is its position, $\mathrm{M}$ is the halo virial mass, and $\mathrm{n}$ is the total particle number within $R_{v i r}$. The centre offset is defined as $\Delta_{r}=\left|\boldsymbol{R}_{c m}-\boldsymbol{R}_{c}\right| / R_{v i r}$. We note here that the density peak position is used as cluster centre $\boldsymbol{R}_{c}$ (see Paper I, for more discussion about different centre definitions).

Velocity dispersion deviation The velocity dispersion $\sigma$ is always an important quantity for cluster dynamics. It is often used to predict the cluster's dynamical mass through the virial theorem:

$\frac{1}{2} M_{\text {total }} \sigma^{2} \propto \frac{G M_{\text {total }}^{2}}{R}$,

where $\mathrm{G}$ is the universal gravitational constant, and $M_{\text {total }}$ and $\mathrm{R}$ are the cluster mass and radius. Thus, one can easily get the predicted dynamical mass through $M_{\text {total }} \propto$ $\left(R \sigma^{2}\right) / G$. However, this is based on the assumption that the cluster is in dynamical equilibrium, which is normally not true. Therefore, we define a parameter to quantify the deviation to the dynamical equilibrium: $\zeta=\frac{\sigma}{\sigma_{t}}$, here $\sigma_{t}=\sqrt{\left(G M_{\text {total }}\right) / R}$. Note that the velocity dispersion deviation $\zeta$ can be different from unity even for perfectly relaxed clusters, because its exact value also depends on the density profile.

\section{RESULTS}

\subsection{Radial profiles}

We first show the radial profiles of these four parameters: $\eta, \zeta, \Delta_{r}$ and $f_{s}$, in Fig. 1 from upper to lower panels respectively. We select three clusters with different $\eta$ values order as in plot $\left(>1, \approx 1\right.$ and $<1$ from the DM run at $\left.R_{v i r}\right)$. The results from all particles are shown with solid lines and filled symbols, while the symbols with dotted lines from only dark matter particles (DMPs). Different colours and symbols styles represent different versions of simulations, which are indicated on the legend of the top middle panel.

It is worth to note at here again that the DM run has two family dark matter particles: the first (more massive) one shares the same mass and ID to the dark matter particle in the two hydro-dynamical runs; while the second family only has its mass the same as the initial gas particles in the two hydro-dynamical runs. We have verified that this separation in our DM run does not show signs of mass segregation. This particular set in the DM run allows us to make equal comparisons to the two hydro-dynamical runs. If it is not particularly noted, the dark matter particle (DMP) from the DM run refers to the first family (heavier) particle in the lower part.

To calculate the values of these four parameters at each radius $R_{i}$, we simply use the corresponding particles within that radius. However, only particles inside the spherical shell $R_{i, 0.8} \leqslant R \leqslant R_{i}$ are used to calculate the surface pressure energy $E_{s, i}$.

(i) At inner region, the values of $\eta$ are all larger than 1 for all three galaxy clusters, which means that the values of $2 T-E_{s}$ are always larger than their potential energy $|W|$. At outer radius (mostly $R \gtrsim 0.6 R_{v i r}$ ), $\eta$ becomes more flat for all three clusters and three runs. $\eta$ from both the CSF run and the AGN run is normally smaller than from the DM run over all radii. However, there is a better agreement between these three runs, when only DMP is taken into account, especially at outer regions. It means that DMPs are less affected by baryons.

(ii) $\zeta$ normally has a value smaller than 1 , and shows a declining trend from inner to outer radii, which is basically the same saw in $\eta$. In agreement with $\eta$, galaxy clusters simulated with baryon models also have smaller $\zeta$ values than the DM run. It not surprising that the result from DMP is also similar to $\eta$. However, there is a slightly larger disagreement between the three runs, especially for the two with $\eta \leqslant 1$.

(iii) The radial profile for the centre-of-mass offset $\Delta_{r}$ shows large difference between the three galaxy clusters and the three simulation versions. As DMPs contribute the largest mass for galaxy clusters, it is not surprising to see that the dotted lines basically follow the solid lines. It seems to have less correlation between $\Delta$ and $\eta$, seeing from these radial profiles. 

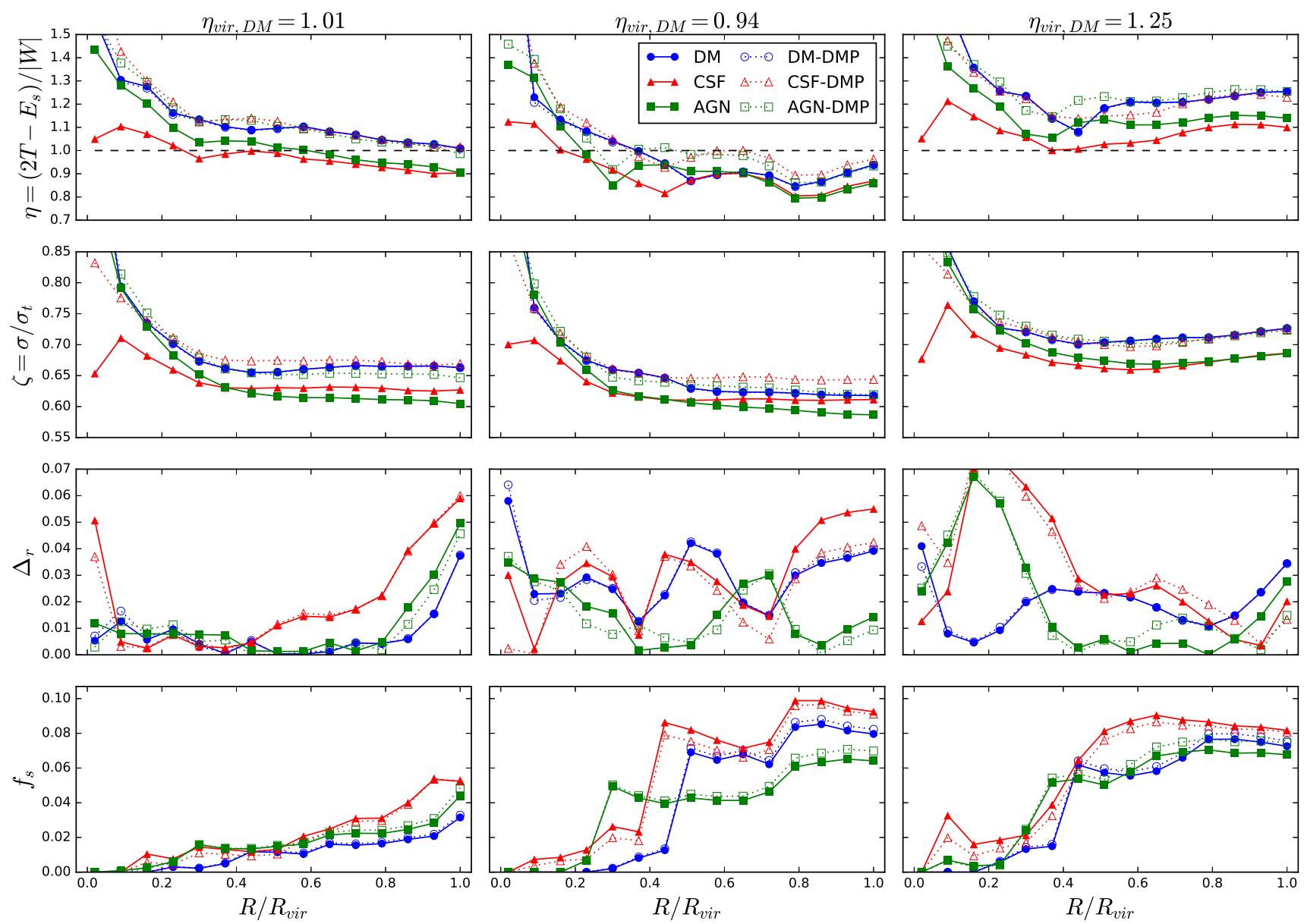

Figure 1. The values of $\eta, \zeta, \Delta_{r}, f_{s}$ (from top to bottom panels) as a function of normalized radius out to $R_{v i r}$. From left to right columns, we show the three example clusters with different $\eta_{D M}$ values at $R_{v i r}: \eta_{v i r, D M}=1.01,0.94,1.25$, respectively. As shown in the legend on the top middle panel, blue filled circles are for the DM run; red filled triangles are for the CSF run; green filled squares are for the AGN run. The corresponding open symbols with dotted lines are the results from their dark matter components. We further note here that the dark matter component in the DM run refer to the first family of dark matter particles (see more details in section 4.1). Solid lines indicate the results from all particles inside the cluster.

(iv) It is not surprising that $f_{s}$ from the CSF run normally has a larger value than the other two runs. This is caused by the over-cooling problem, which affects not only central galaxies, but also satellite galaxies. Similar to $\Delta_{r}$, there is very little differences between the total (solid lines) and DMP (dotted lines) for the $f_{s}$ profile. $f_{s}$ for all three clusters show a clear increasing trend from inner to outer regions. This is simply because the closer to the centre, the higher possibility that substructures are destroyed. This trend is anti-correlated with the radial profile from $\eta$.

Fro these three example clusters, $\eta$ shows a decreasing trend from inner to outer radii, which means that galaxy clusters can be highly un-virialized at their centres than the outer region. In agreement with Shaw et al. (2006), $\eta$ at outer radius $\left(R \gtrsim 0.6 R_{v i r}\right)$ becomes more flat, which means that $\eta$ is primely determined by materials inside $0.6 R_{v i r}$. The in-falling materials at outer region has less effect on $\eta$. It is interesting to see that baryons give a systematic decreasing effects on $\eta$ over the whole radii. However, the $\eta$ from DMP seems to be less affected. Because gravity is the only interaction between dark matter and gas, and gas only occupy a small mass fraction of clusters with a smoother distribution, it not surprising to see this results. Because larger $\sigma$ at fixed radius corresponds to larger $T$, it is also not surprising to see that $\zeta$ basically follows the trend of $\eta$.

There is no clear trend for the profile of $\Delta_{r}$. This is because the centre of mass is largely relying on the mass distributions, especially the substructure position. However, $f_{s}$ shows an increasing trend as radius increases.

\subsection{The baryonic effects}

We further investigate baryon effects on the four parameters in Fig. 2. To explicitly show and understand this effect, changes of these four parameters from the DM run to the two hydro-dynamical runs are separated into two rows: the upper row shows the results from all types of particles; while the lower row is from DMPs. These results are shown as a function of their halo masses $M_{v i r}$. As shown in the legends of right panels, the different color and style symbols indicate different simulations; while the different color and style 

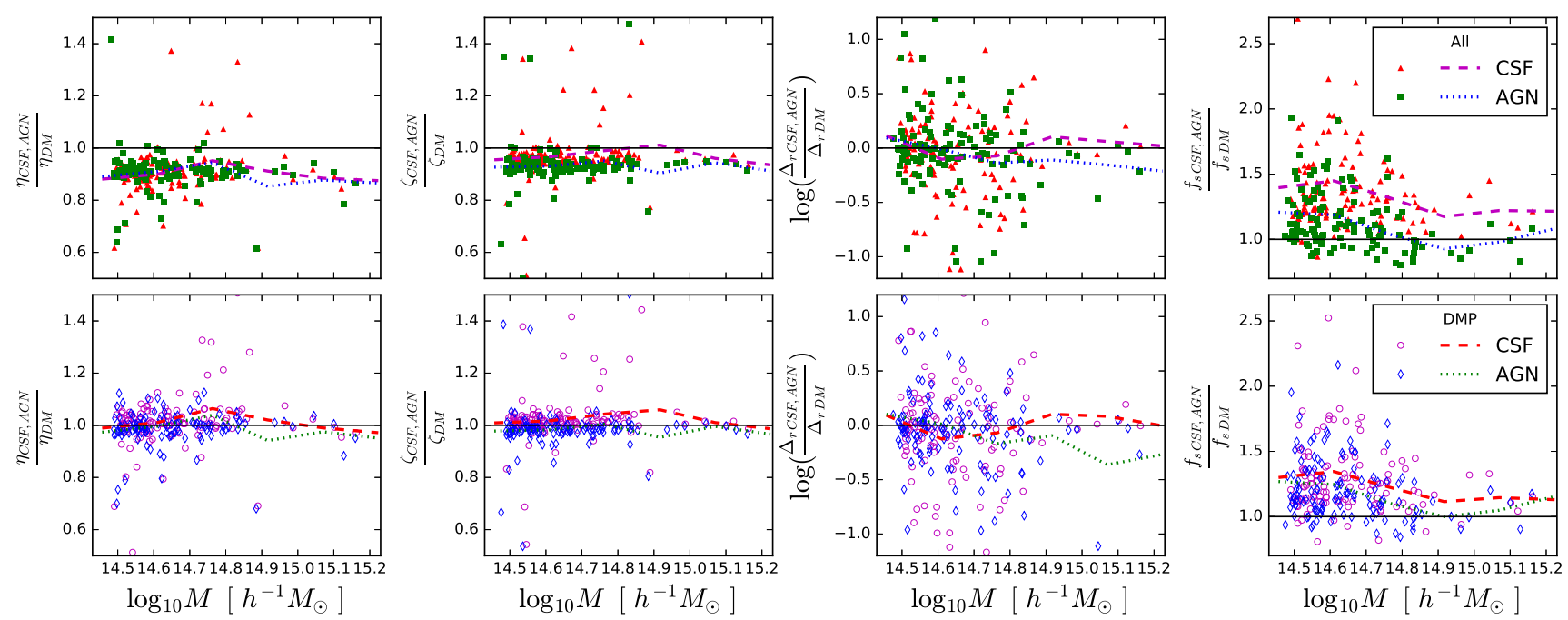

Figure 2. From left to right, ratios, as a function of halo masses between the CSF/AGN run and the DM run for the virial ratio $\eta$, the velocity dispersion deviation $\zeta$, the centre-of-mass offset $\Delta_{r}$ and the substructure mass fraction $f_{s}$, respectively. Lower panels show the same quantities but for DMPs. As indicated in the legends on right panels, red triangles with magenta dashed lines (which are the mean of data points) are coming from the CSF run, while green squares with blue dotted lines indicate the AGN run. The reversed color is used for the results from DMPs as shown in the legend of the lower right panel. Similar to in Fig. 1, DMP in DM run refer to the first family of dark matter particles (see more details in section 4.1; while upper panel show the results including all particles.

lines are the mean of data points. The upper row shows the results from all particles; while the lower row is from DMPs.

Through these comparisons, we find:

(i) The upper panel from the first column shows the ratio of $\eta$, which is calculated with all particles. It is clear that $\eta$ from both the CSF run and the AGN run is about 10 per cent lower than the one from the DM run. Nevertheless, there is very small difference between the two hydro-dynamical runs evident from their mean values. The ratio of $\eta$ shows almost no dependence on cluster masses.

The lower panel shows the results from DMPs. There is almost no difference between the two hydro-dynamical runs and the DM run, which is consistent with the finding from Fig. 1. Although the red dashed line (the CSF run) is on top of the green dotted line (the AGN run), there is very little difference between the CSF run and the AGN run without any dependence on cluster masses.

(ii) We show the ratio of $\zeta$ in the second column of Fig. 2. Again, the mean of $\zeta$ from both the CSF run and the AGN run is slightly lower ( $\sim 0-10$ per cent) than from the DM run. However, $\zeta$ from the AGN run is closer to the $\mathrm{DM}$ results than from the CSF run. Again, this ratio shows almost no dependence on cluster masses.

Similar to the $\eta$ results from DMPs, the mean ratio of $\zeta$ from both the CSF run and the AGN run to the DM run is around 1. The difference between the CSF run and the AGN run is in consistent the result from the upper panel: red dashed line (the CSF run) is always on top of green dotted line (the AGN run).

(iii) The changes of $\Delta_{r}$ are shown in log space in the third column. Due to its sensitivity to the position of substructures, which seems to be easily affected by baryons, there is a large scatter for these data points. However, the mean ratio of $\Delta_{r}$ is around 1 for the CSF run; while this is also true for the AGN run at smaller mass, but a slightly smaller $\Delta_{r}$ than the DM run is shown at larger mass.

Since dark matter normally occupies more than 80 per cent of total cluster mass, it is not surprising that the lower panel, which shows the result from DMPs, gives very similar results as the upper panel.

(iv) We show how $f_{s}$ changes in the last column. $f_{s}$ is clear larger from the CSF run than the DM run: it increases about 40 per cent at smaller cluster mass; it is still about 20 per cent higher at larger cluster mass. $f_{s}$ from the AGN run is 20 per cent lower than from the CSF run; 20 per cent higher than the DM run at smaller cluster masses, and almost no difference between the two runs at $M \gtrsim 10^{14.8} h^{-1} \mathrm{M}_{\odot}$.

For the changes of $f_{s}$ from DMPs in the lower panel, we see very similar result for the AGN run as in the upper panel. Nevertheless, $f_{s}$ from the CSF run is around 10 per cent closer to the DM run than its result in the upper panel over all cluster mass range.

The bottom-left panel in Fig. 2 shows that $\eta$ is consistent for DMPs between the three runs, which implies that baryons have little effect on both the kinetic and potential energy of dark matter if $E_{s}$ is ignored. We find a fixed value of $\eta_{D M P} / \eta_{A l l}$ for all clusters. This value from the two hydrodynamical runs is $\sim 10$ per cent higher than from the DM run. This means that baryons have a systematic change on $\eta$. This is consistent with the finding from the top-left panel of Fig. 2. We study this below.

Similar to the findings from radial profiles in Fig. 1, $\zeta$ also shows the closest correlation with $\eta$ for the change caused by baryons. Although the ratio between $\zeta_{A G N}$ and $\zeta_{D M}$ is very similar to the ratio of $\eta, \zeta_{C S F}$ is much closer to $\zeta_{D M}$ than $\eta_{C S F}$ to $\eta_{D M}$. This shows that the over-cooling problem in the CSF run has more effect on $\zeta$ than $\eta$.

In agreement with Fig. 1, baryons have a large influence on $\Delta_{r}$. It is not surprising that $\Delta_{r, D M P}$ follows $\Delta_{r, A l l}$, and 
both have a large scatter. However, the mean changes of $\Delta_{r}$ seem to rest on 1, except the drop at high mass end from the AGN run. This large scatter can be caused by the sensitivity of the mass distribution to baryons: 1) galaxy cluster centers can be changed from the DM run to the two hydrodynamical runs; 2) the positions and masses of substructures can be altered by baryons.

$f_{s}$ from the AGN run seems to suffer a weak baryon effect, except at smaller mass clusters, which tend to have higher $(\sim 20$ per cent) substructure mass fraction than the DM run, while the over-cooling problem is more obvious for $f_{s}$ : substructures from the CSF run are more massive than from the DM run.

$\eta$ is calculated from kinetic energy $\mathrm{T}$, potential energy $\mathrm{W}$, and surface pressure energy $E_{s}$. In 3 , we study how $\eta$ is derivative to T, W, $E_{s}$. From left to right columns, we show the baryon effects on T, W, $E_{s}$ and $M_{v i r}$ respectively. The upper row shows the results from all particles; while the lower panel results are coming from DMPs.

The key findings of Fig. 3 are summarized as:

(i) The ratio of kinetic energy $\mathrm{T}$ is shown in the first column. Again, the upper panel shows the results from all particles. $T_{C S F} / T_{D M}$ is about 0.95 . However, the mean of this ratio drops to $\sim 0.9$ at both larger and smaller mass end; while it reaches $\sim 1.0$ at $M \approx 10^{14.8} h^{-1} \mathrm{M}_{\odot} . T_{A G N} / T_{D M}$ is about $0.85-0.9$. For the result coming from DMPs on the lower panel, both ratios have a constant shift up of $\sim 10$ per cent.

(ii) The second column shows the ratio of potential energy W. For the results from all particles in the upper panel, $W_{C S F} / W_{D M}$ is about 1.05 , which is gradually reaching 1.0 at the massive mass end. On the contrary, $W_{A G N} / W_{D M}$ is about 0.95 , increasing to $\sim 1.0$ for the most massive clusters. For the results from DMPs on the lower panel, this ratio for the CSF run is almost the same; while the AGN run slightly $(\sim 3$ per cent) shift up.

Although both the CSF and AGN runs tend to have similar virial and dark matter masses as the DM run (actually the total mass from the AGN run is a little lower than the DM run at smaller halo masses, see the fourth column of this figure for more detail), the over-cooling problem in the CSF run tends to result a much higher concentration (see more discussion in Cui et al. 2016a), and so a higher potential energy than the AGN run.

(iii) We show ratios of $E_{s}$ in the third column. We have verified that $E_{s}$ only occupies $\sim 20$ per cent of the total kinetic energy $\mathrm{T}$. It means that $E_{s}$ has a minor contribution to $\eta$. As shown on the upper panel, the baryon effect on the total $E_{s}$ is very similar ( $\sim 5$ per cent lower than the DM run) between the AGN run and the CSF run. It is not surprising that DMPs contribute similar to $E_{s}$ between these three runs, that is shown on the lower panel.

From this we conclude that $E_{s}$ is irresponsible for the baryon effect. The unchanged $\eta_{D M P}$ for the CSF run is because baryons have a similar increasing ( $\sim 5$ per cent) effect on $\mathrm{T}$ and $\mathrm{W}$, while both $\mathrm{T}$ and $\mathrm{W}$ seem to be unaffected by baryons for the AGN run.

For the baryon effect on $\eta_{\text {total }}$, the key difference is in $T_{\text {total }}$. The drops of $T_{\text {total }}$ in both hydro-dynamical runs are possibly caused by collisional gas, of which thermal energy is either dissipated due to turbulences and frictions, or locked up into stars.

\subsection{The classification of relaxed and unrelaxed clusters}

Separating out relaxed clusters from unrelaxed ones is not an easy task. Neto et al. (2007) adopted $2 T /|U|<1.35$, $\Delta_{r}<0.07$ and $f_{s}<0.1$ to select relaxed galaxy clusters. They found $\sim 50$ per cent of halos at $M_{v i r}=10^{15} h^{-1} \mathrm{M}_{\odot}$ are relaxed. However, they did not take the surface pressure energy $E_{s}$ into account in their virial ratio calculation. Shaw et al. (2006) adopted a slightly narrower limit $(\beta=$ 0.2 , equivalent to $|1-\eta|<0.2)$ to select virial equilibrium halos with $E_{s}$ in their $\eta$. With only this criterion, they excluded 3.4 per cent of 2159 halos $\left(M_{\text {halo }} \gtrsim 3 \times 10^{13} h^{-1} \mathrm{M}_{\odot}\right)$ as un-virialized ones. Power et al. (2012) picked out dynamically relaxed halos with a slightly smaller $\Delta_{r}<0.04$ at $z=$ 0 . From this we conclude that there is no consistency in the literature about parameter for relaxed halos.

In Fig. 4, we investigate relations between these parameters: $\eta$ vs $\zeta$ (left column), $\Delta_{r}$ (middle column), $f_{s}$ (right column), which are normally used for classifying cluster dynamical states. From top to bottom, we show results from the AGN, the CSF and the DM runs, respectively. Symbol color encodes the cluster velocity dispersion $\sigma$, indicated in the top colorbar. Dashed vertical lines show $\eta=1$, where clusters are in dynamical equilibrium. Grey regions indicate limits inside which galaxy clusters are relaxed.

In agreement with Fig. 2, there is a good linear correlation between $\eta$ and $\zeta$ shown in the left column of Fig. 4. This is because $\sigma$ in $\zeta$ is equivalent to the square root of $\mathrm{T}$ in $\eta$, while $\sigma_{t}$ is similar to a square root of $\mathrm{W}$. For all three versions of simulations, $\zeta$ is around 0.65 at $\eta=1$. After excluding some noisy data points with $\eta<0.8$, we find very similar slopes after linear fitting. Thus, we simply use all data points at the same time to fit, which results in black dotted lines with a slope of 0.312 . This leads us to propose $\zeta$ as a proxy for $\eta$, which can be deduced from observation. All particles are used to calculate $\sigma$ and $\zeta$ here. Thus, to apply this relation on observations, one needs to consider the bias of using galaxies as the velocity dispersion tracer, which has been investigated and corrected in Munari et al. (2013), while for cluster mass $\mathrm{M}$ in $\sigma_{t}$, one can use lensing mass from observation. Using simulations with mock observation images, Puchwein \& Bartelmann (2007) have shown that the recovered lensing mass does not depend on the assumption of hydrostatic equilibrium. Similar to our proposal, Puchwein \& Bartelmann (2007) also suggested to use the difference between dynamically recovered mass from X-ray and lensing mass to distinguish dynamical states.

Although smaller $\Delta_{r}$ tends to have $\eta$ closer to 1 , there are clusters that have larger $\Delta_{r}$ with $\eta \rightarrow 1$. Similarly the same is true for $f_{s}$. The virial equilibrium implies that $<$ $d^{2} I / d t^{2}>=0$, time-averaged over a period that is long compared to the local dynamical timescale (Shaw et al. 2006; Poole et al. 2006). Therefore, we expect a roughly symmetric distribution around zero due to those halos that are oscillating around the virial equilibrium position. These halos with large $\Delta_{r}$ and $f_{s}$ but $\eta \rightarrow 1$ could be still in the process 

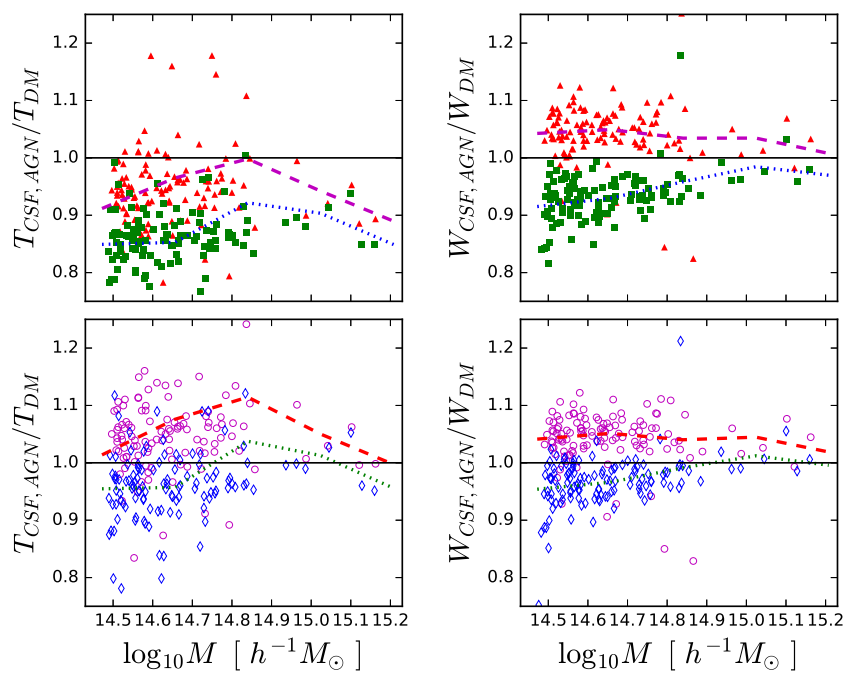
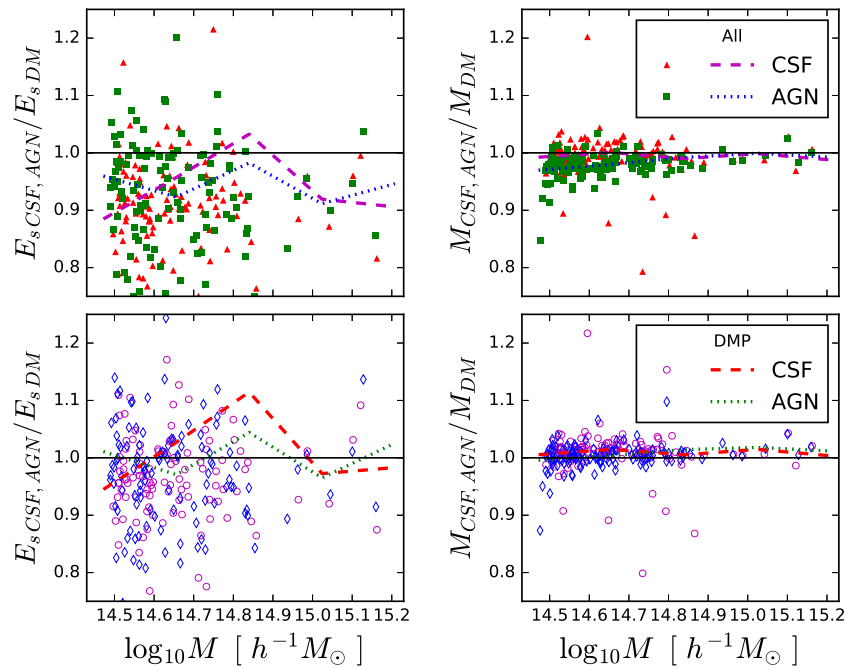

Figure 3. Similar plots as Fig 2, but for kinetic energy T, potential energy W, surface pressure energy $E_{s}$ and galaxy cluster mass $M_{v i r}$. We refer to Fig 2 and the two legends on the right panels for the meanings of the colors, symbols and lines.

of settling down to dynamical relaxation, but with a glimpse of equilibrium.

For our limited cluster mass range, we do not see a clear mass dependence on these parameters in Fig. 4. However, $\sigma$ shows a weak dependence on these parameters, especially in the left column, where higher $\sigma$ value tends to have both higher $\eta$ and $\zeta$ values. However, this trend is not clear for $\Delta_{r}$ and $f_{s}$.

From Fig. 4, there is no bimodal distribution in any of the runs for either single or combined parameters. Data points from all three simulations have a similar distribution, other than a weak decrease of $\eta$ and a weak increase of $f_{s}$ from the DM run to the two hydro-dynamical runs.

Applying the selection criteria from Neto et al. (2007), we find that 70 (78 and 78) out of 123 halos from the DM run (from the CSF run and the AGN run, respectively) are dynamically relaxed. This gives a similar relaxation fraction as Neto et al. (2007). One can visually find out that most of unrelaxed clusters are cut out by limits from $\Delta_{r}$ and $f_{s}$, which is also in agreement with Neto et al. (2007). Power et al. (2012) suggested a smaller value of $\Delta_{r} \approx 0.04$ to select dynamically relaxed halos (see also Macciò et al. 2007). Observational results suggest a much lower relaxation fraction: $\sim 28$ per cent from SDSS survey (Wen \& Han 2013); 16 per cent from X-ray selected clusters (Mantz et al. 2015). Thus, we apply restricted criteria to select out relaxed clusters: $0.85<\eta<1.15$ (Knebe \& Power 2008); $\Delta<0.04$ (Power et al. 2012); $f_{s}<0.075$. By applying these thresholds, we select out 41,43 and 48 dynamical relaxed clusters from the DM, the CSF and the AGN runs respectively. This gives a relaxation fraction of $\sim 35$ per cent. $29(\sim 65$ per cent) of these relaxed clusters are cross identified in all three runs; 34 ( $\sim 75$ per cent) of them are cross identified in both the CSF and the AGN runs. In agreement with the baryon effect on individual parameters, most of halos have their dynamical relaxation states unchanged. Although AGN feedback impacts on substructures as well as $f_{s}$, it plays a minor role in changing the dynamical state of clusters.

\section{DISCUSSION AND CONCLUSIONS}

Using our simulated galaxy cluster catalogue of 123 galaxy clusters from Paper I, we investigated the dynamical state of clusters in the DM (dark-matter-only) run; the CSF (gas cooling, star formation and supernova feedback) run and the AGN (with also AGN feedback) run. These three sets of simulations allow us to explore how baryons affect cluster dynamical states. We examined four parameters: the virial ratio $\eta$, the velocity dispersion deviation $\zeta$, the centre of mass offset $\Delta_{r}$ and the substructure mass fraction $f_{s}$, which are normally used to separate dynamically relaxed clusters from unrelaxed ones.

The main results are summarised as follows.

(i) The radial profiles of $\eta$ and $f_{s}$ become relatively constant at outer radius $\left(R \gtrsim 0.6 R_{v i r}\right)$. However, $\Delta_{r}$ does not show such features. It means that we can expect $\eta_{500} \approx \eta v i r$ and $f_{s, 500} \approx f_{s, v i r}$. However, this is not applicable for $\Delta_{r}$.

(ii) The baryon models (both with and without AGN feedback) have a weak effect on $\eta$, which is $\sim 10$ per cent lower in the two hydro-dynamical compared to the DM run. This is mainly caused by the drop of kinetic energy $\mathrm{T}$ with gas dynamics. Therefore, $\eta_{D M P}$ shows very similar results between all three runs.

Baryon models have no impact on $\Delta_{R}$ for the CSF run; this is also true for the AGN run at smaller masses, but there is a slightly smaller $\Delta_{r}$ in the AGN run than in the DM run at the higher mass end.

$f_{s}$ is about 40 (20) per cent higher in the CSF run than in the DM run at smaller (higher) masses, while $f_{s}$ from the AGN run is 20 per cent lower than from the CSF run.

(iii) There is good linear correlation between $\eta$ and $\zeta$ for all three runs, which encourages us to use $\zeta$ as an indicator of $\eta$, which can not be easily measured from observation. Using this relation, one can deduce the virial ratio for observed galaxies.

(iv) For all the investigated parameters, there is no clear bimodal distribution between relaxed and unrelaxed clusters. 

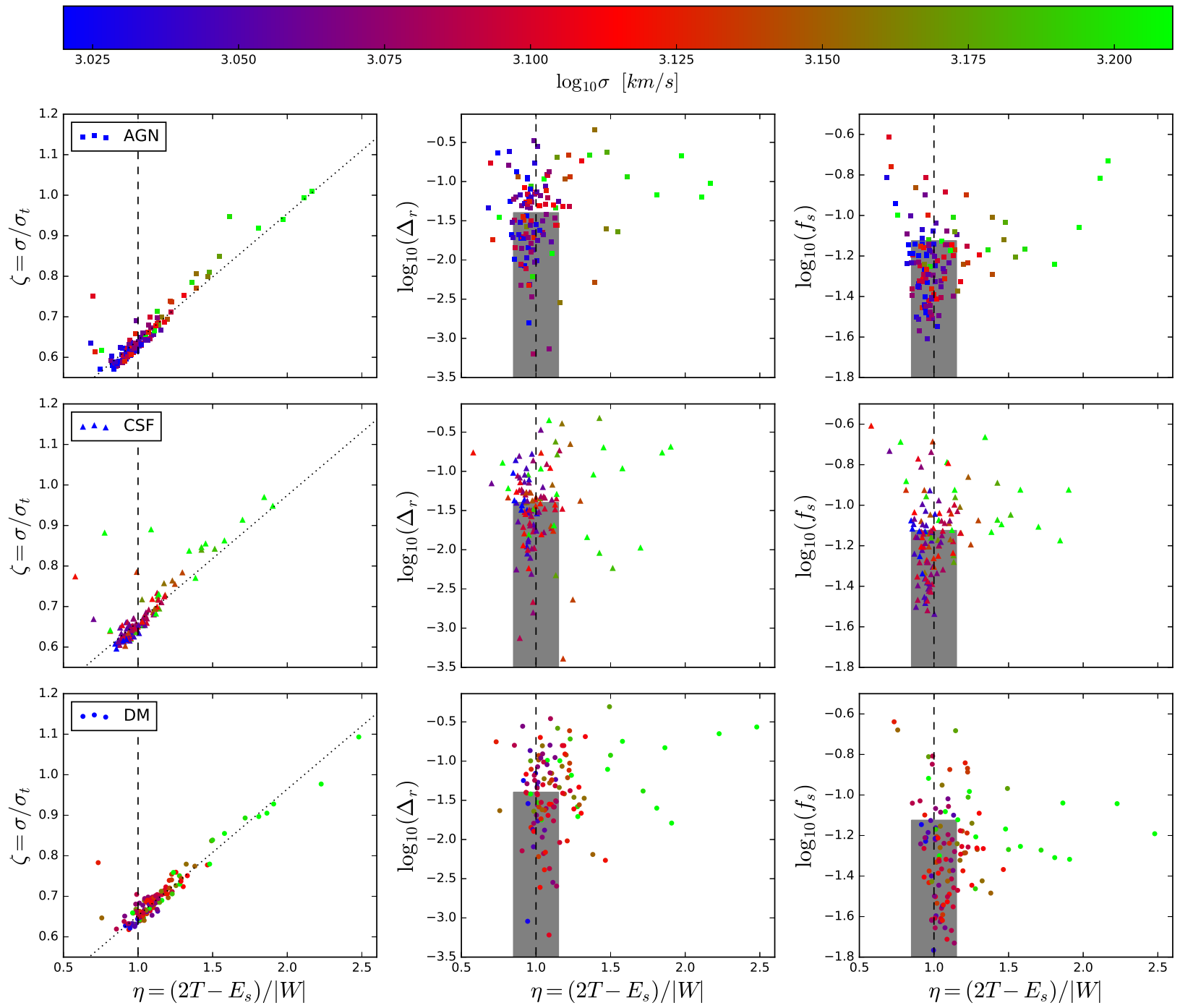

Figure 4. Relations between $\zeta$ (left column), $\Delta_{r}$ (middle column) $f_{s}$ (right column) with the virial ratio $\eta$. From top to bottom panels, we show results from the AGN, the CSF and the DM runs. Symbol color is coding to its velocity dispersion $\sigma$, which is shown by the colorbar on the top of this plot. Dashed vertical lines indicate $\eta=1$, where the cluster is in dynamical equilibrium. Dotted lines on the left column are fitting results to data points with a fix slope of 0.312 . Galaxy clusters located inside grey regions in the middle and right columns can be classified as relaxed clusters.

(v) With more restricted thresholds for $\eta, \Delta_{r}$ and $f_{s}$, we find that $\sim 35$ per cent of our sample clusters are relaxed, in which $\sim 65$ per cent are cross identified in all three runs. This means that baryons play a minor role in regulating cluster dynamical states.

Using controlled cluster simulations, Poole et al. (2006) quantified the effects of mergers on the dynamical state of galaxy clusters and showed that dark matter typically relaxes slightly later than gas. A recent work by Zhang et al. (2016), who also used controlled cluster simulations but only with adiabatic gas, investigated baryon effects on merger times. They found merger timescale can be shortened by a factor of up to 3 for clusters with gas fractions of 0.15 , compared to the one without gas. This indicates that clusters with baryons will virialize faster than ones without baryons, which is similar to the finding in Poole et al. (2006). With galaxy clusters from cosmology simulations, we only find that baryons decrease the virial ratio by $\sim 10$ per cent from the DM run, which makes the mean of $\eta$ in the two hydrodynamical runs much closer to 1 . Because clusters in cosmological volume can never be isolated because mergers and in-falling material are ongoing, their dynamical states can hardly be exactly in dynamical relaxed. We further note here that the relaxation fraction seems to be unaffected $(\lesssim 5$ per cent) by baryons. This could because 1 ) our cluster sample is not large enough; 2) this relaxation fraction depends on the arbitrary selection limits. The total baryon mass fraction is normally around $10-15$ per cent within galaxy clusters (e.g. Sun 2012; Gonzalez et al. 2013; Lagana et al. 2013; Borgani et al. 2006; Planelles et al. 2013). It is interesting 
to see that $\eta$ is dragged down around a similar fraction by baryons, while its value from dark matter component is almost untouched. Another unchanged quantity is the linear relation between $\eta$ and $\zeta$, which urges us to propose a simple fitting function for observers to get $\eta$ from observed galaxy clusters. However, there is no bimodal distribution between relaxed and unrelaxed galaxy clusters. It makes a tough task for choosing the limits for these parameters to select out galaxy clusters in dynamical equilibrium.

Using different wavelength tracers to determine dynamical states of galaxy clusters can give different answers. Using photometric data of the Sloan Digital Sky Survey, Wen \& Han (2013) derived the asymmetry, the ridge flatness and the normalized deviation of a smoothed optical map, which is coming from the brightness distribution of member galaxies. With their defined relaxation parameter from the upper three quantities, they found that 28 per cent of 2092 clusters are dynamically relaxed. In X-ray observation, the power ratio and the centroid shift are normally used to select out dynamically relaxed clusters (e.g. Böhringer et al. 2010; Rasia et al. 2013). In addition, Mantz et al. (2015) proposed the symmetry-peakiness-alignment criterion for classifying cluster dynamical states. With their criterion, they report a relaxation fraction of 16 per cent for their $361 \mathrm{X}$-ray selected clusters. Combining different wavelength results could give accurate answers. For example, Ge et al. (2016) has investigated the dynamical state of two paired clusters under optical, X-ray and radio emissions; Rossetti et al. (2016) characterized the dynamical states of galaxy clusters detected with the Sunyaev-Zeldovich (SZ) effect by the Planck and compare them with their dynamical states derived from Xray surveys. They found a slightly higher relaxation fraction from the X-ray sample ( $\sim 74$ per cent) than from SZ sample $(\sim 52$ per cent), which could due to different selection effects.

The reliability and agreement between these tracers from different wavelength observations, between different methods, as well as the consistency with theoretical predictions are still unclear. We will address these questions with our galaxy cluster sample in the next paper.

\section{ACKNOWLEDGEMENTS}

All the figures in this paper are plotted using the python matplotlib package (Hunter 2007). This research has made use of NASA's Astrophysics Data System (ADS), the arXiv preprint server and Wikipedia. Simulations have been carried out at the CINECA supercomputing Centre in Bologna, with CPU time assigned through ISCRA proposals and through an agreement with the University of Trieste. WC acknowledges the supports from University of Western Australia Research Collaboration Awards PG12105017, PG12105026, from the Survey Simulation Pipeline (SSimPL; http://www.ssimpl.org/) and from iVEC's Magnus supercomputer under National Computational Merit Allocation Scheme (NCMAS) project gc6. WC, CP, AK, GFL, and GP acknowledge support of ARC DP130100117. CP, AK, and GFL acknowledge support of ARC DP140100198. CP acknowledges support of ARC FT130100041. SB and GM acknowledge support from the PRIN-INAF12 grant 'The Universe in a Box: Multi-scale Simulations of Cosmic Struc- tures', the PRINMIUR 01278X4FL grant 'Evolution of Cosmic Baryons', the INDARK INFN grant and 'Consorzio per la Fisica di Trieste'. AK is supported by the Ministerio de Economia y Competitividad (MINECO) in Spain through grant AYA2012-31101 as well as the Consolider-Ingenio 2010 Programme of the Spanish Ministerio de Ciencia e Innovación (MICINN) under grant MultiDark CSD2009-00064. He further thanks Luna for lunapark. GP acknowledges support from the ARC Laureate program of Stuart Wyithe.

\section{REFERENCES}

Bett P., Eke V., Frenk C. S., Jenkins A., Helly J., Navarro J., 2007, MNRAS, 376, 215

Böhringer H., et al., 2010, A\&A, 514, A32

Borgani S., et al., 2006, MNRAS, 367, 1641

Bryan G. L., Norman M. L., 1998, ApJ, 495, 80

Chandrasekhar S., 1961, Hydrodynamic and hydromagnetic stability

Cui W., Borgani S., Dolag K., Murante G., Tornatore L., 2012, MNRAS, 423, 2279

Cui W., et al., 2014a, MNRAS, 437, 816

Cui W., Borgani S., Murante G., 2014b, MNRAS, 441, 1769

Cui W., et al., 2016a, MNRAS,

Cui W., et al., 2016b, MNRAS, 456, 2566

Davis A. J., D'Aloisio A., Natarajan P., 2011, MNRAS, 416, 242

Dolag K., Borgani S., Murante G., Springel V., 2009, MNRAS, 399, 497

Ge C., Wang Q. D., Tripp T. M., Li Z., Gu Q., Ji L., 2016, MNRAS, 459, 366

Gonzalez A. H., Sivanandam S., Zabludoff A. I., Zaritsky D., 2013, e-prints arXiv:1309.3565,

Hunter J. D., 2007, Computing In Science \& Engineering, 9, 90

Jing Y. P., 2000, ApJ, 535, 30

Klypin A., Yepes G., Gottlöber S., Prada F., Heß S., 2016, MNRAS, 457, 4340

Knebe A., Power C., 2008, ApJ, 678, 621

Knebe A., Libeskind N. I., Knollmann S. R., Yepes G., Gottlöber S., Hoffman Y., 2010, MNRAS, 405, 1119

Lagana T. F., Martinet N., Durret F., Lima Neto G. B., Maughan B., Zhang Y.-Y., 2013, A\&A, 555, A66

Ludlow A. D., Navarro J. F., Li M., Angulo R. E., Boylan-Kolchin M., Bett P. E., 2012, MNRAS, 427, 1322

Macciò A. V., Dutton A. A., van den Bosch F. C., Moore B., Potter D., Stadel J., 2007, MNRAS, 378, 55

Mantz A. B., et al., 2015, MNRAS, 446, 2205

Mo H., van den Bosch F. C., White S., 2010, Galaxy Formation and Evolution

Munari E., Biviano A., Borgani S., Murante G., Fabjan D., 2013, MNRAS, 430, 2638

Navarro J. F., Frenk C. S., White S. D. M., 1997, ApJ, 490, 493

Neto A. F., et al., 2007, MNRAS, 381, 1450

Planelles S., Borgani S., Dolag K., Ettori S., Fabjan D., Murante G., Tornatore L., 2013, MNRAS, 431, 1487

Poole G. B., Fardal M. A., Babul A., McCarthy I. G., Quinn T., Wadsley J., 2006, MNRAS, 373, 881

Power C., Knebe A., Knollmann S. R., 2012, MNRAS, 419, 1576

Puchwein E., Bartelmann M., 2007, A\&A, 474, 745

Rasia E., Meneghetti M., Ettori S., 2013, The Astronomical Review, 8, 40

Rossetti M., et al., 2016, MNRAS, 457, 4515

Schaller M., et al., 2015, MNRAS, 452, 343

Shaw L. D., Weller J., Ostriker J. P., Bode P., 2006, ApJ, 646, 815

Springel V., 2005, MNRAS, 364, 1105

Springel V., White S. D. M., Tormen G., Kauffmann G., 2001, Monthly Notices of the Royal Astronomical Society, 328, 726 
Sun M., 2012, New Journal of Physics, 14, 045004

Velliscig M., van Daalen M. P., Schaye J., McCarthy I. G., Cacciato M., Le Brun A. M. C., Dalla Vecchia C., 2014, MNRAS, 442, 2641

Velliscig M., et al., 2015, preprint, (arXiv:1504.04025)

Wen Z. L., Han J. L., 2013, MNRAS, 436, 275

Zhang C., Yu Q., Lu Y., 2016, ApJ, 820, 85

van Daalen M. P., Schaye J., Booth C. M., Dalla Vecchia C., 2011, MNRAS, 415, 3649

This paper has been typeset from a $\mathrm{T}_{\mathrm{E}} \mathrm{X} / \mathrm{L}_{\mathrm{A}} \mathrm{T}_{\mathrm{E}} \mathrm{X}$ file prepared by the author. 